Republic of China); and science and its social relations (Prof. A. R. Wadia). Among the special lectures, the following may be mentioned: synthesis and stereochemistry of carotenoids (Prof. Paul Karrer); metallogenetic provinces and epochs in Japan (Prof. T. Watanabe); extensive showers of cosmic rays (Prof. P. Auger); iron and manganese resources of Japan (Prof. T. Watanabe); present state of geological education in Japan (Prof. T. Watanabe); structure of proteins (Prof. Linus Pauling); sode ssh industry (Dr. T. P. Hou); and Chinese herbal medicines (Dr. Hsieh Yu).

The meetings of the Executive Committee and of the Council of the Indian Science Congress Association were held on January 3. The meeting of the General Committee was held on January 8, at which it was announced that Dr. B. C. Roy was elected as general president for the session 1957, that is, for the year 1956-57. As announced last year, Dr. M. S. Krishnan, director of the Geological Survey of India, will be general president for the year 1955-56. The next session of the Indian Science Congress will be held in Agra in 1956.

\section{THE MASS SPECTROSCOPE}

$T$ HE handbook, prepared by M. G. Inghram of the University of Chicago, and R. J. Hayden of the Argonne National Laboratory, for the Subcommittee on Instruments and Techniques of the Committee on Nuclear Science of the National Research Council*, presents in some detail the fundamental design and operational techniques pertinent to the efficient utilization of the mass spectroscope. Attention is directed mainly to the ionic type of mass analyser with either electrical or photographic ionic detection. No attempt is made to discuss the wide range of application of mass spectroscopes or to include particulars of the associated electronic circuits, but adequate references to articles on these topics are given.

The handbook consists of four sections. In the first the equations appropriate to the various types of analysers are stated and explained. They relate mainly to instruments with focusing properties. A table, in which some seventy different instruments grouped according to type and field arrangement are listed, follows. The theories to be applied to all instruments of a given type are similar, and the major differences are largely of constructional detail, some of which, together with the names of the constructors of the instruments and dates of publication, are specified in the trable or discussed at length in the text. The second section is devoted to the important problem of suitable ion sources, and the various types are classified according to their primary uses and their sensitivities. A table is included showing the compounds appropriate for analysis of each of the elements up to bismuth from gaseous, crucible or surface ionization sources, together with an indication of the preferred source. In the third section brief consideration is given to the problems of sample introduction into the ionization region of the mass spectrometer where, unless special precautions are

* National Academy of Sclences: National Research Council. Nuclear Science Serios-Report No. 4: Mass Spectroscopy. By Mark G. Inghram and Richard J. Hayden. (Prepared for the Subcommittee on Instruments and Techniques of the Committee on Nuclear Science, Division of Physical Sciences, National Research Council.) Pp. iv 51. (Washington, D.C.: National Acaderny of Sciences, 1954.) taken, the relative intensity of the ion beams formed by the source will not be characteristic of the sample.

The final section deals with ion detectors, and in addition to the conventional photographic plate and electrometer the use of photomultipliers and scintillation counters is discussed and the design and construction of several types of detectors are outlined and shown schematically.

The handbook concludes with a bibliography of more then two hundred references, and though these refer to dates earlier than April 1952 when the manuscript was completed, the report as a whole represents a most valuable source of information on basic principles and procedures of mass spectroscopy. S. WEINTRoUB

\section{THE GLOBAL RADIATION IN A RAYLEIGH ATMOSPHERE}

\section{By DIRAN DEIRMENDJIAN and ZDENEK SEKERA \\ University of California, Los Angeles, California}

$\mathrm{T}$

THE publication of Chandrasekhar's treatise, "Radiative Transfer", 1 marked an important step in the exact treatment of radiation problems in a scattering medium. One such problem, the complete quantitative description of the radiation field in a scattering atmosphere according to the laws of Rayleigh, was solved as an illustration of the possibilities of the new method. Its success has already been demonstrated by Chandrasekhar and Elbert ${ }^{2}$ in explaining the main characteristics of the sky-light polarization observed on clear days.

Another application of the method consists of finding the total flux of energy, as a function of the wave-length and direction of the unpolarized light, received at the lower boundary of a plane-parallel scattering atmosphere of finite depth illuminated by parallel radiation of a given flux from a single direction. Under certain assumptions, in the case of the terrestrial atmosphere this constitutes what is known as the 'global radiation'. It can be shown ${ }^{3}$ that the relative global radiation, that is, that arising from an incident flux of unit strength at the corresponding wave-length, can be derived from Chandrasekhar's discussion, in which the independent variables are the optical thickness (defining the wavelength) and the parameter $\mu_{0}=\cos \boldsymbol{\theta}_{0}$, where $\boldsymbol{\theta}_{0}$ is the zenith distance of the sun. The resulting expression is a very simple one in terms of the functions $\gamma l$ and $\gamma_{r}$, which are identicel with those mentioned in equations (7) and (8) of ref. 2. We have then :

$$
G\left(\tau, \mu_{0}\right)=\frac{1}{2} \mu_{0}\left[\gamma_{l}\left(\tau, \mu_{0}\right)+\gamma_{\gamma}\left(\tau, \mu_{0}\right)\right],
$$

in which $G\left(\tau, \mu_{0}\right)$ represents the relative global radiation, that is, the flux of the sun and sky radiation through a horizontal surface, resulting from the multiple scattering of the incident light. The functions $\gamma z\left(\tau, \mu_{0}\right)$ and $\gamma_{r}\left(\tau, \mu_{0}\right)$ give the individuel contributions of the components of the incident light polarized in directions parallel and normal to the vertical plane through the sun respectively, and their sum,

$$
\frac{Q\left(\tau, \mu_{0}\right)}{\mu_{0}}=\frac{1}{2}\left[\gamma l\left(\tau, \mu_{0}\right)+\gamma_{r}\left(\tau, \mu_{0}\right)\right]
$$

